\title{
Growth of the phase change enthalpy induced by the crystal transformation of an inorganic-organic eutectic mixture of magnesium nitrate hexahydrate-glutaric acid
}

Suimin Li $i^{a}$, Shao Lin ${ }^{a}$, Ziye Ling ${ }^{a, b *}$, Xiaoming Fang ${ }^{a, b}$, Zhengguo Zhang ${ }^{a, b *}$

${ }^{a}$ Key Laboratory of Enhanced Heat Transfer and Energy Conservation, The Ministry of Education, School of Chemistry and Chemical Engineering, South China University of Technology, Guangzhou 510640, China.

${ }^{\mathrm{b}}$ Guangdong Engineering Technology Research Center of Efficient Heat Storage and Application, South China University of Technology, Guangzhou 510640, China.

E-mail: zyling@scut.edu.cn ; cezhang@scut.edu.cn

Tel.:+86 208711 2845; Fax: +862087113870 


\section{Uncertainties calculation of DSC}

Type A uncertainty is the statistical uncertainty component, calculated by the equation given as Eq. (1):

$$
\mu_{A}=\sqrt{\frac{\sum\left(x_{i}-\bar{x}\right)^{2}}{(n-1) n}}
$$

Where, $\mu_{A}$ is the Type A uncertainty, $x_{i}$ is the experimental value for each time, $\bar{x}$ is the average of all values, $\mathrm{n}$ is the number of experiment times.

Type B uncertainty is the non-statistical uncertainty component, usually dependent on the instrument error and calculated by the equation given as Eq. (2):

$$
\mu_{B}=\frac{\Delta_{\text {instrument }}}{\sqrt{3}}
$$

Where, $\mu_{B}$ is the Type B uncertainty, $\Delta_{\text {instrument }}$ is the instrument error. The uncertainty of the DSC in $\mathrm{T}$ and $\Delta \mathrm{H}$ correspond to $1.0^{\circ} \mathrm{C}$ and $2.0 \%$.

The final uncertainty is calculated by $\mu_{A}$ and $\mu_{B}$ according to the equation given as Eq. (3):

$$
\mu=\sqrt{\mu_{A}^{2}+\mu_{B}^{2}}
$$

The uncertainties calculation results are shown in Table 1 and Table 2.

Table 1 The uncertainties of MNH- GA binary in melting point and the melting enthalpy.

\begin{tabular}{llll}
\hline $\mathbf{w t} \%$ & $\mathbf{T}_{\mathbf{m}} /{ }^{\circ} \mathrm{C}$ & $\triangle \mathbf{H}_{\mathbf{m}} /\left(\mathbf{J} \cdot \mathbf{g}^{-1}\right)$ & $\boldsymbol{\mu}_{\Delta \mathbf{H m}} / \%$ \\
\hline $\mathbf{1 0 0}$ & $89.9 \pm 0.6$ & $165.2 \pm 3.7$ & 2.2 \\
$\mathbf{7 0}$ & $67.3 \pm 0.6$ & $176.6 \pm 4.3$ & 2.4 \\
$\mathbf{6 5}$ & $66.5 \pm 0.6$ & $179.4 \pm 5.5$ & 3.1 \\
$\mathbf{6 0}$ & $66.7 \pm 0.6$ & $189.0 \pm 2.3$ & 1.2 \\
$\mathbf{5 5}$ & $67.0 \pm 0.6$ & $186.5 \pm 3.7$ & 2.0 \\
$\mathbf{5 0}$ & $66.9 \pm 0.6$ & $186.9 \pm 3.7$ & 2.0 \\
$\mathbf{4 0}$ & $65.9 \pm 0.9$ & $193.8 \pm 2.6$ & 1.3 \\
$\mathbf{0}$ & $97.3 \pm 0.7$ & $175.3 \pm 4.2$ & 2.4 \\
\hline
\end{tabular}


Table 2 The uncertainties of MNH- GA, MNH- GA/EG and MNH- GA/EP in melting point and the melting enthalpy before and after cycling.

\begin{tabular}{llll}
\hline Samples & $\mathbf{T}_{\mathbf{m}} /{ }^{\circ} \mathbf{C}$ & $\mathbf{H}_{\mathbf{m}} /\left(\mathbf{J} \cdot \mathbf{g}^{-1}\right)$ & $\boldsymbol{\mu}_{\triangle \mathbf{H m}} / \%$ \\
\hline MNH-GA & $66.7 \pm 0.6$ & $189.0 \pm 2.3$ & 1.2 \\
MNH-GA/EG & $63.5 \pm 0.7$ & $75.7 \pm 3.4$ & 4.5 \\
MNH-GA/EP & $65.1 \pm 0.6$ & $102.3 \pm 7.3$ & 7.1 \\
MNH-GA/EP-without cover (100 cycles) & $63.3 \pm 0.6$ & $59.9 \pm 3.5$ & 5.9 \\
MNH-GA/EP-with cover (100 cycles) & $62.3 \pm 0.6$ & $100.0 \pm 1.6$ & 1.6 \\
\hline
\end{tabular}

2. Video recording of crystallization process

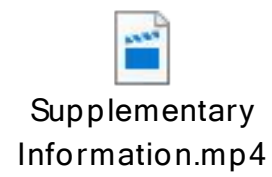

Video 1 The crystallization process video of $60 \mathrm{wt} \% \mathrm{MNH}-\mathrm{GA}$ eutectic mixture. 